\title{
Consumo e digestibilidade da matéria seca de fenos de braquiária decumbens e amendoim forrageiro em ovinos estimados por meio de $n$-alcanos ${ }^{1}$
}

\author{
Nelson Massaru Fukumoto ${ }^{2}$, Julio Cesar Damasceno ${ }^{3}$, Cristiano Côrtes ${ }^{4}$, Rafael Cesário \\ Paine $^{5}$, Maria Fernanda Soares Queiroz ${ }^{5}$, Geraldo Tadeu dos Santos ${ }^{3}$, Makoto Matsushita 6 \\ 1 Parte da dissertação de Mestrado do primeiro autor apresentada à Universidade Estadual de Maringá. Pesquisa financiada pelo CNPq. \\ 2 Doutorando em Zootecnia - DZO/UEM. \\ ${ }^{3}$ Departamento de Zootecnia - UEM, Avenida Colombo, 5790, CEP: 87020-900, Maringá - PR. Bolsista do CNPq. \\ 4 Departamento de Zootecnia - UEPG/UFPR. \\ 5 Zootecnista - UEM. \\ 6 Departamento de Química da UEM.
}

RESUMO - O experimento foi realizado com os objetivos de avaliar a técnica de $n$-alcanos para estimar o consumo e a digestibilidade da MS em ovinos e definir a estratégia de coleta de fezes e os alcanos internos a serem utilizados no cálculos. Vinte ovinos foram distribuídos em delineamento inteiramente casualizado e alimentados com dietas formuladas com diferentes proporções de amendoim forrageiro (Arachis pintoi Koprov \& Gregory cv. Amarillo) e braquiária decumbens [Urochloa decumbens(Stapf), Webster] (0, 15, 30, 45 e 60\% de amendoim forrageiro, base da MS). O período experimental teve duração de 18 dias, dez de adaptação à dieta, três para administração do indicador externo $\left(\mathrm{C}_{32}\right.$ em péletes de celulose $)$ e cinco de coleta de fezes. As fezes foram coletadas seis vezes ao dia $(9,13,17,21,1$ e $5 \mathrm{~h})$, diretamente no reto do animal. Os dados foram submetidos à análise de variância e as comparações das médias estimadas aos valores reais foram feitas utilizando-se o teste $\mathrm{t} a$ 5\% de significância. Para determinação da digestibilidade aparente da MS, os animais foram equipados com bolsas coletoras de fezes. O consumo foi calculado utilizando-se os alcanos internos $\mathrm{C}_{31}$ e $\mathrm{C}_{33}$ isoladamente ou em conjunto. Para o cálculo da digestibilidade da MS, foram utilizados os alcanos internos $\mathrm{C}_{33}$ a $\mathrm{C}_{35}$. Para obtenção de estimativas adequadas de digestibilidade, é necessária a correção para os valores de recuperação fecal dos indicadores internos, observando-se que os melhores resultados foram obtidos quando se utilizou o alcano interno $\mathrm{C}_{33}$ nos cálculos. Para estimar o consumo de MS, devem-se utilizar, de forma conjunta nos cálculos, os alcanos internos $\mathrm{C}_{31} \mathrm{eC}_{33}$, sem necessidade de correção para recuperação fecal, podendo-se coletar uma única amostra de fezes por animal por dia, pela manhã (9 h) ou à tarde (13 h).

Palavras-chave: cera cuticular, cromatografia gasosa, dotriacontano, indicadores, recuperação fecal, ruminantes

\section{Use of $n$-alkanes to estimate intake and digestibility of signalgrass and forage peanut in sheep}

\begin{abstract}
The objective of this trial was to use n-alkanes to estimate dry matter intake (DMI) and DM digestibility in sheep. It was also of particular interest to compare two methodologies of fecal collection (total collection vs. grab samples) as well as to identify which n-alkanes best estimate DMI and DM digestibility. Twenty animals were assigned to a completely randomized design and were fed diets containing different proportions of forage peanut (Arachis pintoi Koprov \& Gregory cv. Amarillo) and signalgrass hays [Brachiaria decumbens, (Stapf), Webster] (0, 15, 30, 45 and 60\% of forage peanut; DM basis). The experiment lasted 18 days with 10 days for diet adaptation, three days for marker administration $\left(\mathrm{C}_{32}\right.$ in cellulose pellets), and five days for fecal collection. Data were submitted to analysis of variance and observed and estimated means were compared by $t$ test. Fecal grab samples were collected six times daily at 4 hours intervals (9:00, 13:00, 17:00, 21:00, 01:00 and 05:00) for five consecutive days. The internal alkanes $\mathrm{C}_{31}, \mathrm{C}_{33}$, and $\mathrm{C}_{31}+\mathrm{C}_{33}$ were used for estimating DMI while DM digestibility was estimated by $\mathrm{C}_{33}, \mathrm{C}_{35}$, and $\mathrm{C}_{33}+\mathrm{C}_{35}$. Results showed that reliable estimation of DM digestibility was obtained by using the internal alkane $\mathrm{C}_{33}$ corrected for fecal recovery. The combination of the internal alkanes $\mathrm{C}_{31}+\mathrm{C}_{33}$ resulted in the best estimation of DMI with no need for fecal recovery correction. In addition, only one daily fecal grab sample (9 h or $13 \mathrm{~h}$ ) was necessary to accurately estimate DMI.
\end{abstract}

Key Words: cuticular wax, dotriacontane, fecal recovery, gas chromatography, markers, ruminant 


\section{Introdução}

O conhecimento sobre a forragem consumida pelo animal em pastejo é importante, principalmente em países tropicais, onde a pecuária tem como base as pastagens. Desse modo, espera-se que a quantidade de forragem consumida, aliada à sua qualidade, satisfaça totalmente ou em grande parte as exigências de mantença, crescimento e produção dos animais (Silva \& Nascimento Jr., 2001).

Ao estimar o consumo e a composição da dieta consumida, é possível aplicar as exigências nutricionais da espécie e da categoria animal a um desempenho diário esperado e fazer inferências até que ponto os diferentes alimentos utilizados são capazes de suprir essas necessidades, permitindo uma alimentação econômica e nutricionalmente correta (Oliveira, 2002).

A avaliação do valor alimentício de forragens fornecidas aos ruminantes envolve conhecimento do consumo e da digestibilidade. No entanto, a maioria destes estudos é conduzida sob condições controladas, em experimentos convencionais de digestibilidade, onde é feita a coleta total de fezes, tendo-se um controle da quantidade consumida. Como este tipo de ensaio envolve custos, muito trabalho e tempo e as medidas obtidas sob condições controladas não podem ser extrapoladas para condições de pastejo, o uso de $n$-alcanos tem se destacado.

Para que o uso de $n$-alcanos na estimativa do consumo, da composição e da digestibilidade dos animais em pastejo s eja realizado com precisão, são necessárias informações exatas quanto ao modo de dosagem do alcano externo, à forma e freqüência de amostragem de fezes, às análises laboratoriais destas amostras e aos procedimentos de cálculos.

Apesar de o uso de $n$-alcanos ser relativamente recente, encontram-se disponíveis vários trabalhos com informações importantes sobre a aplicabilidade desta metodologia em estudos com gramíneas e leguminosas de clima temperado (Dove, 1992; Mayes \& Duncan, 1999; Côrtes et al., 2005a). No entanto, são escassos e recentes os trabalhos com informações sobre a possibilidade de utilização da técnica de $n$-alcanos em experimentos com forrageiras de clima tropical (Côrtes et al., 2005a, b).

Alguns pesquisadores (Mayes et al., 1986; Dove et al., 1989; Marais et al., 1996) têm demonstrado preocupação em desenvolver métodos que facilitem a dosagem do alcano externo e a coleta de amostras sem comprometimento nas estimativas. Neste contexto, experimentos para estudo das frequiências de amostragem de fezes em condições tropicais são necessários para aumentar a praticidade e a confiabilidade nos dados, determinar melhor os padrões de trânsito gastrointestinal e excreção do alcano e reduzir os custos na utilização da técnica.

Assim, este trabalho foi realizado com os objetivos de avaliar a técnica de $n$-alcanos para estimar o consumo e a digestibilidade da MS de forragens tropicais em ovinos, definir a estratégia da amostragem de fezes e escolher os alcanos internos a serem utilizados nos procedimentos de cálculos, por meio de experimento em gaiolas para estudos de metabolismo.

\section{Material e Métodos}

O experimento foi realizado no setor de Ovinocultura e Caprinocultura da Fazenda Experimental de Iguatemi (FEI), pertencente à Universidade Estadual de Maringá (UEM/ Maringá-PR).

Foram utilizados 20 ovinos machos, sem raça definida $(24,59 \pm 2,78 \mathrm{~kg}$ de $\mathrm{PV})$, em delineamento experimental inteiramente casualizado, com quatro repetições por tratamento. As dietas fornecidas aos animais foram compostas de diferentes proporções de fenos de amendoim forrageiro (Arachis pintoi Koprov \& Gregory cv. Amarillo) ebraquiária decumbens (Urochloa decumbens, Stapf, Webster) (0, $15,30,45$ e $60 \%$ de amendoim forrageiro, com base na MS). $\mathrm{O}$ feno de amendoim forrageiro foi confeccionado no Instituto Agronômico do Paraná (IAPAR - Estação Experimental de Londrina) e o de braquiária no campus da Universidade Estadual de Maringá (UEM). O capim e o amendoim forrageiro foram picados grosseiramente e posteriormente misturados e homogeneizados nas diferentes proporções para compor as dietas experimentais.

Os animais foram everminados e alojados em baias individuais com piso ripado e suspenso, com livre acesso ao bebedouro e ao cocho, onde foi fornecido sal mineral à vontade.

O período experimental foi de 18 dias, dez de adaptação dos animais à dieta fornecida, três de adaptação para estabilização do alcano externo no trato digestório do animal e cinco de coleta de fezes.

Durante o período experimental, os animais foram alimentados duas vezes ao dia (8 e 16h), à vontade, permitindo-se aproximadamente $15 \%$ de sobras. A dieta fornecida e as sobras foram devidamente pesadas por animal e por dia.

O alcano externo (sintético) administrado aos animais foi o $\mathrm{C}_{32}$ (dotriacontano 97\% - Acros Organics), utilizando-se como veículo o papel de celulose. As cápsulas foram confeccionadas conforme descrito por Mayes et al. (1986). Folhas de celulose puras foram cortadas em tamanho de 
17 x 17 cm e colocadas em bandeja de alumínio (descartável). Em um béquer de $200 \mathrm{~mL}$, foi adicionada $(150 \mathrm{~mL})$ uma solução de heptano $\left(\mathrm{C}_{7} \mathrm{H}_{16}\right)$ e alcano $\mathrm{C}_{32}$ aquecida a $80^{\circ} \mathrm{C}$. A solução continha $1,8 \mathrm{~g}$ do alcano $\mathrm{C}_{32}$, suficiente para preparar nove cápsulas de $200 \mathrm{mg}$. Na bandeja, as folhas de celulose foram revolvidas para que houvesse absorção uniforme da solução. Depois de volatilizado todo o heptano, as folhas foram retiradas da bandeja e secas no interior da capela. Em seguida, foram colocadas por 5 minutos em estufa a $105^{\circ} \mathrm{C}$ para melhor impregnação do alcano na celulose. Após o resfriamento, as folhas foram peletizadas com uso de uma prensa, em formato cilíndrico de tamanho $1,5 \times 2,0 \mathrm{~cm}$ (diâmetro x altura) para facilitar a dosagem ao animal. Os péletes, contendo $200 \mathrm{mg}$ de alcano $\mathrm{C}_{32}$, foram administrados aos animais oralmente uma vez ao dia (às $8 \mathrm{~h}$ ).

As fezes, aproximadamente $20 \mathrm{~g}$, foram coletadas seis vezes ao dia, em intervalo de 4 horas $(9,13,17,21,1$ e 5 h), durante cinco dias, diretamente do reto do animal. Para o cálculo da produção fecal, os animais foram equipados com bolsas coletoras adaptadas ao dorso. As bolsas foram esvaziadas duas vezes ao dia.

Durante o período de coleta de fezes, também foram amostrados, diariamente, os fenos fornecidos. As amostras de fezes e dos fenos foram armazenadas em freezera- $10^{\circ} \mathrm{C}$. Posteriormente, foram secas em estufa de ventilação forçada a $55^{\circ} \mathrm{C}$ por 72 horas, sendo moídas em peneira de $1 \mathrm{~mm}$ para posteriores análises.

Utilizando-se as amostras de fezes, com base na quantidade de MS de cada dia, formaram-se amostras compostas (dos 5 dias de coleta) por animal e por horário. Foram realizadas ainda amostras compostas dos fenos dos cinco dias de amostragem.

A extração dos $n$-alcanos foi realizada no Laboratório de Nutrição Animal do Departamento de Zootecnia (DZO) da UEM. Os $n$-alcanos foram analisados conforme procedimentos descritos por Côrtes et al. (2005a). A técnica baseia-se no processo de saponificação direta das amostras. Amostras de 1,5 $\mathrm{g}$ de feno e de $0,5 \mathrm{~g}$ de fezes foram pesadas em balança analítica e moídas em peneira de $1 \mathrm{~mm}$. Nos frascos de $50 \mathrm{~mL}$, com tampa de rosca, foram adicionados 0,3016 mg de tetratriacontano $99,97 \%\left(\mathrm{C}_{34} \mathrm{H}_{70}-\right.$ Sigma Aldrich), diluídos em $4 \mathrm{~mL}$ de $n$-heptano $95 \%\left(\mathrm{C}_{7} \mathrm{H}_{16}\right)$ a cada amostra como padrão interno. Evaporado o $n$-heptano, as amostras foram acrescidas de $14 \mathrm{~mL}$ de solução alcoólica de hidróxido de potássio ( $\mathrm{KOH}$ a 1,5 M) e incubadas em banho-maria a $90^{\circ} \mathrm{C}$ sob agitação durante 4,5 horas. Depois de resfriadas à temperatura ambiente, foram adicionados $20 \mathrm{~mL}$ de heptano e $10 \mathrm{~mL}$ de água destilada, mantendo-se a mistura a $60^{\circ} \mathrm{C}$ sob agitação vigorosa durante 15 minutos. Após a decantação (aproximada- mente 1 hora), o sobrenadante foi coletado com uma pipeta de pasteur em tubos de ensaios e parcialmente evaporados. Em seguida, o conteúdo foi purificado em colunas de sílica-gel (200 - 400 mesh - Acros Organics) e suspenso em heptano com volume de leito de $10 \mathrm{~mL}$. A mistura heptano $+n$-alcanos foi recuperada em balões de $50 \mathrm{~mL}$, sendo evaporado todo heptano à temperatura ambiente, ficando somente os hidrocarbonetos sólidos aderidos.Posteriormente, os $n$-alcanos foram rediluídos em microtubos de $2 \mathrm{~mL}$ com $1,5 \mathrm{~mL}$ de $n$-heptano $95 \%\left(\mathrm{C}_{7} \mathrm{H}_{16}\right)$.

A identificação dosn-alcanos das amostras foi realizada por cromatografia gasosa no Laboratório de Alimentos do Departamento de Química (DQI) da UEM.

Foi utilizado cromatógrafo a gás SHIMADZU 14, com coluna capilar de sílica fundida (OV-5 de $30 \mathrm{~m}$ x 0,32 mm e $0,25 \mu \mathrm{m}$ de espessura de filme) e detector de ionização de chama (FID). Os fluxos dos gases ultrapuros (White Martins), foram de 1,2 mL.min ${ }^{-1}$ para o gás de arraste $\left(\mathrm{H}_{2}\right), 30 \mathrm{~mL}^{\mathrm{min}}{ }^{-1}$ para o gás auxiliar (Make-up) $\left(\mathrm{N}_{2}\right)$ e 30 e $300 \mathrm{~mL}^{-\mathrm{min}^{-1}}$ para os gases de chama $\mathrm{H}_{2}$ e ar sintético, respectivamente.

As temperaturas do injetor (INLET) e detector (FID) foram de 300 e $315^{\circ} \mathrm{C}$, respectivamente. A coluna foi operada com temperatura inicial de $220^{\circ} \mathrm{C}$ por 2 minutos, com aumento na razão de $5^{\circ} \mathrm{C} /$ minuto até $297^{\circ} \mathrm{C}$. O modo de injeção foi Split (1:100), sendo a amostra $(3,0 \mu \mathrm{L})$ injetada manualmente com microsseringa de $10 \mu \mathrm{L}$.

As áreas dos picos cromatográficos correspondentes a cada $n$-alcano foram determinadas por meio de um Integrador-Processador CG-300, no qual a identificação dos $n$-alcanos de comprimento de cadeia $\mathrm{C}_{24} \mathrm{aC}_{36}$ baseou-se na comparação ao padrão (Acros Organics), pelo tempo médio de retenção, contendo os alcanos tetracosano $\left(\mathrm{C}_{24} \mathrm{H}_{50}\right)$, hexacosano $\left(\mathrm{C}_{26} \mathrm{H}_{54}\right)$, dotriacontano $\left(\mathrm{C}_{32} \mathrm{H}_{66}\right)$, tetratriacontano $\left(\mathrm{C}_{34} \mathrm{H}_{70}\right)$ e hexatriacontano $\left(\mathrm{C}_{36} \mathrm{H}_{74}\right)$. Posteriormente, foram convertidas as quantidades de $n$-alcanos por referência ao padrão interno $\mathrm{C}_{34} \mathrm{H}_{66}(0,3016$ $\mathrm{mg}$ /amostra) e calculados para quilograma de MS para a amostra considerada.

O consumo médio por animal foi calculado utilizando-se, separadamente, os indicadores internos $\mathrm{C}_{31}$ e $\mathrm{C}_{33}$ e a média das concentrações dos alcanos $\mathrm{C}_{31}+\mathrm{C}_{33}$ presentes nas fezes e na dieta fornecida, por meio da equação proposta por Mayes et al. (1986):

$$
\text { Consumo animal }(\mathrm{kgMS} / \mathrm{dia})=\frac{(I F x E D)}{[(I P x E F)-(I F x E P)]}
$$

em que: IF = alcano interno presente nas fezes $(\mathrm{mg} / \mathrm{kg}$ de $\mathrm{MS}) ; \mathrm{ED}=$ alcano externo dosado ( $\mathrm{mg} / \mathrm{dia}) ; \mathrm{IP}=$ alcano 
interno presente no feno $(\mathrm{mg} / \mathrm{kg}$ de $\mathrm{MS}) ; \mathrm{EF}=$ alcano externo presente nas fezes $(\mathrm{mg} / \mathrm{kg}$ de $\mathrm{MS}) ; \mathrm{EP}=$ alcano externo presente no feno ( $\mathrm{mg} / \mathrm{kg}$ de MS).

Para o cálculo da digestibilidade aparente da MS $\left(\mathrm{CDMS}_{\mathrm{AP}}\right)$, utilizou-se a equação:

$$
\mathrm{CDMS}_{\mathrm{AP}}(\%)=[(\mathrm{MSi}-\mathrm{MSe}) / \mathrm{MSi}] \times 100 \text {, }
$$

em que: $\mathrm{CDMS}_{\mathrm{AP}}=$ coeficiente de digestibilidade aparente da MS; $\mathrm{MSi}=$ MS ingerida MSe $=$ MS excretada.

Na digestibilidade da MS estimada pelos alcanos, adotou-se a equação proposta por Mayes \& Lamb (1984) utilizando-se dois alcanos internos separadamente $\left(\mathrm{C}_{33} \mathrm{e}\right.$ $\mathrm{C}_{35}$ e a média de $\mathrm{C}_{33}+\mathrm{C}_{35}$ ):

$$
\mathrm{CDMS}_{\text {Alcano }}(\%)=[1-(\mathrm{ID} / \mathrm{IF})] \times 100 \text {, }
$$

em que: $\mathrm{CDMS}_{\mathrm{Alcano}}(\%)=$ coeficiente de digestibilidade da MS pelo alcano; ID = alcano interno presente na dieta $(\mathrm{mg} / \mathrm{kgMS}) ; \mathrm{IF}=$ alcano interno presente nas fezes $(\mathrm{mg} / \mathrm{kgMS})$.

Para o cálculo da recuperação fecal dos alcanos $\left(\mathrm{C}_{24}\right.$ a $\mathrm{C}_{36}$ ), foram utilizadas as quantidades de alcano consumido e excretado nas fezes no período:

$$
\begin{aligned}
\mathrm{RF}(\%)= & {[\text { alcano excretado }(\mathrm{mg}) / \text { alcano }} \\
& \text { consumido }(\mathrm{mg})] \times 100
\end{aligned}
$$

Os resultados foram submetidos à análise de variância pelo programa computacional SAEG 5.0 (Euclydes, 1993).

Para o cálculo das concentrações dos alcanos considerando as proporções de amendoim forrageiro na dieta, foi realizada análise de regressão e, para comparar as concentrações dos alcanos da dieta, foi utilizado o teste Tukey $(\mathrm{P}<0,05)$.
Os valores digestibilidade e consumo de MS estimados por meio dos alcanos foram comparados aos observados (reais) utilizando-se o teste t a 5\% de significância. As diferenças entre os valores reais e os estimados, bem como o coeficiente de variação das estimativas, também foram utilizadas como parâmetro de avaliação da técnica (Willians, 1993; Detmann et al., 2001; Macedo et al., 2001). O mesmo procedimento foi realizado para determinar o melhor horário de coleta e o número de coletas para estimar o consumo.

A recuperação fecal dos alcanos foi determinada por meio de análise de regressão da recuperação fecal em função do comprimento de cadeia carbônica.

\section{Resultados e Discussão}

$\mathrm{O}$ perfil das concentrações dos $n$-alcanos $\mathrm{C}_{24}$ a $\mathrm{C}_{36}$ das dietas fornecidas aos ovinos é representado na Tabela 1. As extrações das amostras de fenos sem o padrão interno $\left(\mathrm{C}_{34}\right)$ comprovaram que o alcano $\mathrm{C}_{34}$ não foi identificado nas análises e, portanto, não há necessidade da correção deste alcano das amostras com o uso do padrão interno.

Verificou-se predomínio dos alcanos de cadeias ímpares sobre os pares, sendo que as concentrações dos alcanos de cadeia longa, principalmente o $\mathrm{C}_{31}$ e o $\mathrm{C}_{33}$, foram maiores $(\mathrm{P}<0,05)$ e com menores coeficientes de variação. Não houve efeito ( $\mathrm{P}>0,05)$ para os alcanos $\mathrm{C}_{24}, \mathrm{C}_{25}, \mathrm{C}_{26}, \mathrm{C}_{28} \mathrm{e}$ $\mathrm{C}_{32}$ em relação à proporção de amendoim forrageiro na

\begin{tabular}{|c|c|c|c|c|c|c|c|c|c|}
\hline \multirow[t]{2}{*}{$\begin{array}{l}N \text {-alcanos } \\
N \text {-alkanes }\end{array}$} & \multicolumn{6}{|c|}{$\begin{array}{c}\text { Nível de amendoim forrageiro na dieta }(\%) \\
\text { Proportion of dietary forage peanut }(\%)\end{array}$} & \multirow[t]{2}{*}{$\mathrm{CV}(\%)^{3}$} & \multirow[t]{2}{*}{$\begin{array}{c}\text { Efeito }^{4} \\
\text { Effect }\end{array}$} & \multirow[t]{2}{*}{$\begin{array}{l}\text { Média } \\
\text { Mean }\end{array}$} \\
\hline & 0 & 15 & 30 & 45 & 60 & $100^{2}$ & & & \\
\hline $\mathrm{C}_{24}$ & 16,0 & 16,0 & 16,1 & 16,1 & 16,2 & 16,3 & 14,4 & NS & $16,1^{\mathrm{e}}$ \\
\hline $\mathrm{C}_{25}^{24}$ & 5,4 & 5,7 & 6,0 & 6,2 & 6,5 & 7,2 & 22,8 & NS & $6,2^{\mathrm{e}}$ \\
\hline $\mathrm{C}_{26}^{25}$ & 9,4 & 8,9 & 8,5 & 8,0 & 7,6 & 6,4 & 22,8 & NS & $8,1^{\mathrm{e}}$ \\
\hline $\mathrm{C}_{27}^{20}$ & 10,7 & 9,8 & 8,9 & 8,0 & 7,1 & 4,7 & 21,1 & LIN & $8,2^{\mathrm{e}}$ \\
\hline $\mathrm{C}_{28}^{21}$ & 75,1 & 68 & 60,9 & 53,7 & 46,6 & 27,6 & 34,0 & NS & $55,3^{\mathrm{c}}$ \\
\hline $\mathrm{C}_{29}^{28}$ & 38,7 & 36,0 & 33,2 & 30,5 & 27,8 & 20,4 & 17,8 & LIN & $31,1^{\text {de }}$ \\
\hline $\mathrm{C}_{30}$ & 10,7 & 9,7 & 8,8 & 7,9 & 6,9 & 4,4 & 10,8 & LIN & $8,1^{\mathrm{e}}$ \\
\hline $\mathrm{C}_{31}$ & 107,1 & 98,5 & 90,1 & 81,6 & 73,1 & 50,5 & 15,1 & LIN & $83,5^{b}$ \\
\hline $\mathrm{C}_{32}$ & 8,0 & 10,8 & 13,7 & 16,7 & 19,6 & 27,3 & 15,4 & NS & $16,1^{\mathrm{cde}}$ \\
\hline $\mathrm{C}_{33}$ & 197,9 & 178,5 & 159,2 & 139,8 & 120,5 & 68,8 & 9,9 & LIN & $144,1^{\mathrm{a}}$ \\
\hline $\mathrm{C}_{35}^{33}$ & 52,7 & 47,6 & 42,6 & 37,5 & 32,4 & 18,8 & 7,5 & LIN & $38,6^{\mathrm{cd}}$ \\
\hline $\mathrm{C}_{36}$ & 8,6 & 8,1 & 7,7 & 7,3 & 6,8 & 5,6 & 13,9 & LIN & $7,4^{\mathrm{e}}$ \\
\hline Total & 540,0 & 497,8 & 455,5 & 413,2 & 371,0 & 258,2 & 14,4 & LIN & 422,6 \\
\hline
\end{tabular}
dieta. Observou-se efeito linear negativo $(\mathrm{P}<0,05)$ do

Tabela 1 - Concentração de $n$-alcanos (mg/kgMS) em fenos de Brachiaria decumbens e amendoimforrageiro fornecidos a ovinos Table 1 - Concentration of $n$-alkanes ( $\mathrm{mg} / \mathrm{kg} \mathrm{DM}$ ) in signalgrass and forage peanut hays fed to sheep

${ }^{1}$ Alcano $\mathrm{C}_{34}$ não identificado nas amostras extraídas sem o padrão interno $\left(C_{34}\right.$ alkane was not identified in samples extracted without the internal standard).

2 Não fornecida aos ovinos (Not fed to the animals).

${ }^{3} \mathrm{CV}(\%)=$ coeficiente de variação (Coefficient of variation).

${ }^{4}$ Efeito = LIN (Linear), NS (não-significativo) (Effect $=$ LIN [Linear]), NS [not significant]).

${ }^{5}$ Médias seguidas de letras diferentes na coluna diferem $(P<0,05)$ pelo teste Tukey (Means, within a row, followed by different letters are different $(P<0.05)$ by Tukey test). 
aumento de amendoim forrageiro na dieta sobre as concentrações dos alcanos $\mathrm{C}_{27}, \mathrm{C}_{29}, \mathrm{C}_{30}, \mathrm{C}_{31}, \mathrm{C}_{33}, \mathrm{C}_{35}$ e $\mathrm{C}_{36}$.

Concentrações superiores a $50 \mathrm{mg} / \mathrm{kg}$ MS (Laredo et al., 1991; Chen et al., 1998) para os alcanos $\mathrm{C}_{31}$ e $\mathrm{C}_{33}$ demonstram a possibilidade de utilização desses alcanos nas estimação do consumo a partir da equação proposta por Mayes et al. (1986).

Na Tabela 2, são descritos os valores das recuperações fecais dos alcanos $\mathrm{C}_{24}$ a $\mathrm{C}_{36}$. Houve efeito linear $(\mathrm{P}<0,05)$ do aumento da cadeia carbônica dos $n$-alcanos sobre a recuperação fecal, efeito associativo importante, comprovado pelo elevado coeficiente de correlação $(r=0,93)$. Em ovinos alimentados com Lolium perene, Mayes et al. (1986) encontraram valores semelhantes para os alcanos $\mathrm{C}_{27}$ a $\mathrm{C}_{35}$. Os valores foram: $\mathrm{C}_{27}=71,3 ; \mathrm{C}_{28}=76,8$; $\mathrm{C}_{29}=74,5 ; \mathrm{C}_{30}=82,0 ; \mathrm{C}_{31}=85,4 ; \mathrm{C}_{32}=88,9 ; \mathrm{C}_{33}=89,1 \mathrm{e}$ $\mathrm{C}_{36}=93,1 \%$. A correlação entre a recuperação fecal e o comprimento de cadeia carbônica encontrada por esses autores foi de $\mathrm{r}=0,96$. Verifica-se, portanto, que, à medida que aumenta o comprimento da cadeia carbônica, decresce a diferença na recuperação fecal entre os alcanos adjacentes, possibilitando o uso destes alcanos nos cálculos do consumo.

Segundo Newman et al. (1995), a incompleta recuperação fecal dos $n$-alcanos ocasiona subestimativa da digestibilidade e do consumo de MS e, nestes casos, são necessárias correções nas análises. Dove \& Mayes (1991) afirmam que, quando os alcanos naturais e fornecidos possuem recuperações fecais semelhantes, o uso de alcanos adjacentes permite estimativas precisas sem a necessidade de correções.

Entretanto, Oliveira et al. (2000) citaram alguns fatores que podem ocasionar erros nos cálculos da recuperação fecal. Entre eles, o processo de amostragem das fezes e da dieta fornecida, erros laboratoriais e variação individual na absorção parcial dos alcanos pelo trato digestivo do animal.

Na Figura 1 são apresentadas as curvas de excreção fecal dos alcanos $\mathrm{C}_{31}$ a $\mathrm{C}_{35}$, utilizados nas estimativas do consumo e da digestibilidade, em um período de 24 horas. Observou-se variação nas concentrações de todos os alcanos, principalmente daquele administrado $\left(\mathrm{C}_{32}\right)$. As amostras obtidas às 5 e às $21 \mathrm{~h}$ apresentaram as maiores concentrações dos alcanos $\mathrm{C}_{32}$ e $\mathrm{C}_{33}$ nas fezes. Nos horários entre 9 e $17 \mathrm{~h}$, verificou-se menor variação da concentração do alcano administrado, que se apresentou estável, indicando os melhores horários de coleta.

Segundo Mayes et al. (1988), a variação da concentração do alcano administrado $\left(\mathrm{C}_{32}\right)$ é maior que a dos alcanos naturais (internos), visto que os naturais estão aparentemente associados à fase particulada (sólida) da digesta,
Tabela 2 - Médias e erro-padrão para a recuperação fecal dos $n$-alcanos $\mathrm{C}_{24}$ a $_{36}$ em ovinos alimentados com fenos de amendoim forrageiro e Brachiaria decumbens

Table 2 - $\quad M e a n s$ and standard error for fecal recovery of the $n$-alkanes $C_{24}$ to $C_{36}$ in sheep fed diets with different proportions of signalgrass and forage peanut

\begin{tabular}{lc}
\hline$N$-alcanos & $\begin{array}{c}\text { Recuperação fecal }(\%)^{2} \\
\text { Fecal recovery }\end{array}$ \\
\hline $\mathrm{C}_{24}$ & 57,42 \\
$\mathrm{C}_{25}$ & 61,93 \\
$\mathrm{C}_{26}$ & 64,78 \\
$\mathrm{C}_{27}$ & 69,98 \\
$\mathrm{C}_{28}$ & 72,47 \\
$\mathrm{C}_{29}$ & 73,67 \\
$\mathrm{C}_{30}$ & 82,37 \\
$\mathrm{C}_{31}$ & 84,98 \\
$\mathrm{C}_{32}$ & 88,23 \\
$\mathrm{C}_{33}$ & 89,45 \\
$\mathrm{C}_{35}$ & 92,70 \\
$\mathrm{C}_{36}$ & 94,04 \\
$\mathrm{EP}($ SE) & 2,49 \\
$\mathrm{r}^{4}$ & 0,93 \\
\hline
\end{tabular}

${ }^{1}$ Alcano $C_{34}$ utilizado como padrão interno $\left(C_{34}\right.$ alkane was used as internal standard).

2 Efeito linear $(Y=-17,169+3,1968 X), R^{2}=0,87$ (Linear effect, $Y=-17.169+$ 3.1968X), $\left.R^{2}=0.87\right)$.

${ }^{3} \mathrm{EP}=$ erro-padrão $(\mathrm{n}=20)(S E=$ standard error $[n=20])$.

${ }^{4} \mathrm{r}=$ coeficiente de correlação, significativo $(\mathrm{P}<0,05)(r=$ correlation coefficient, significant $[P<0.05])$

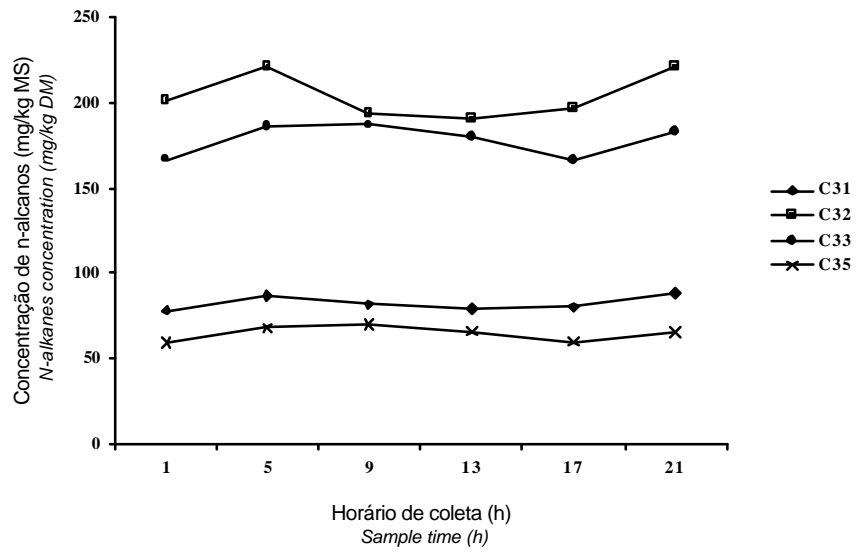

Figura 1 - Excreção dos $n$-alcanos naturais e administrados $\left(\mathrm{C}_{32}\right)$ utilizados nas estimativas de consumo e digestibilidade da MS.

Figure 1 - Fecal excretion of internal and external $\left(C_{32}\right)$ n-alkanes used for estimating of DMI and DM digestibility.

enquanto o administrado apresenta-se de 30 a $40 \%$ na fase líquida, que, em comparação à fase sólida, passa mais rapidamente pelo trato gastrintestinal. Apesar do comportamento diferenciado do alcano administrado e do natural no trato gastrintestinal, as estimativas são válidas desde que a recuperação fecal do par de alcano avaliado seja semelhante (Dove \& Mayes, 1991). Ressalta-se também que as amostragens de fezes foram realizadas diretamente do 
reto do animal, sempre no mesmo horário e com as mesmas quantidades de fezes durante 5 a 7 dias, para obtenção de uma amostra composta ao final do período (Vulich et al., 1995).

Os valores médios da digestibilidade aparente (coleta total de fezes) e da digestibilidade estimada a partir dos $n$-alcanos, seus coeficientes de variação, as diferenças nas estimativas (digestibilidade aparente menos estimada pelos alcanos) e seus respectivos erros-padrão são descritos na Tabela 3.

Os valores estimados da digestibilidade da MS utilizando-se os alcanos internos $\mathrm{C}_{33}$ e $\mathrm{C}_{35}$ separadamente e a média da concentração destes alcanos, todos corrigidos para recuperações fecais, não diferiram estatisticamente $(\mathrm{P}>0,05)$ pelo teste $t$ entre os alcanos utilizados e quando comparados à digestibilidade aparente determinada pela coleta total. Entretanto, sem a correção para a recuperação fecal dos alcanos, os coeficientes de digestibilidade foram subestimados, verificando-se diferença significativa $(\mathrm{P}<0,05)$ entre as digestibilidades estimadas pelos alcanos e a digestibilidade pela coleta total.

Tabela 3 - Digestibilidade aparente e estimada pelosn-alcanos, diferença nas estimativas e erros-padrão da média $^{1,2}$

Table 3 - Values of measured and estimated (n-alkanes) digestibility of $D B=M$, means difference, and standard error of mean

\begin{tabular}{lccc}
\hline $\begin{array}{l}\text { Item }^{1,2} \\
\text { Item }^{1,2}\end{array}$ & $\begin{array}{c}\text { Média } \\
\text { Mean }\end{array}$ & $\mathrm{CV}(\%)^{3}$ & $\begin{array}{c}\mathrm{DM}^{4} \\
M D^{4}\end{array}$ \\
\hline $\mathrm{CDMS}_{\mathrm{AP}}$ & $50,15^{\mathrm{a}}$ & 10,13 & - \\
$\mathrm{CDMS}_{\mathrm{C} 33}$ & $41,50^{\mathrm{d}}$ & 7,49 & $8,65^{\mathrm{a}}$ \\
$\mathrm{CDMS}_{\mathrm{C} 35}$ & $43,69^{\mathrm{b}}$ & 9,00 & $6,46^{\mathrm{c}}$ \\
$\mathrm{CDMS}_{\mathrm{MC} 33 \mathrm{C} 35}$ & $42,59^{\mathrm{c}}$ & 7,04 & $7,55^{\mathrm{b}}$ \\
$\mathrm{CDMS}_{\mathrm{C} 33 \text { cor }}$ & $48,11^{\mathrm{a}}$ & 5,36 & $2,04^{\mathrm{d}}$ \\
$\mathrm{CDMS}_{\mathrm{C} 35 \text { cor }}$ & $48,54^{\mathrm{a}}$ & 7,09 & $1,60^{\mathrm{d}}$ \\
$\mathrm{CDMS}_{\mathrm{MC} 33 \mathrm{C} 35 \text { cor }}$ & $48,33^{\mathrm{a}}$ & 5,59 & $1,82^{\mathrm{d}}$ \\
$\mathrm{EP}^{5}(S E)$ & 0,78 & - & 1,02 \\
\hline
\end{tabular}

${ }^{1} \mathrm{CDMS}_{\mathrm{AP}}=$ coeficiente de digestibilidade aparente da MS, $\mathrm{CDMS}_{\mathrm{C} 33}=$ coeficiente de digestibilidade da MS com $\mathrm{C}_{33}, \mathrm{CDMS}_{\mathrm{C} 35}=$ coeficiente de digestibilidade da MS com $\mathrm{C}_{35}, \mathrm{CDMS}_{\mathrm{MC} 33 \mathrm{C} 35}=$ média de coeficiente de digestibilidade da MS com $\mathrm{C}_{33}+\mathrm{C}_{35}$, $\mathrm{CDMS}_{33 \mathrm{cor}}=$ coeficiente de digestibilidade da MS com $\mathrm{C}_{33}$ corrigida, $\mathrm{CDMS}_{\mathrm{C} 35 \mathrm{cor}}=$ coeficiente de digestibilidade da MS com $\mathrm{C}_{35}$ corrigida, $\mathrm{CDMS}_{\mathrm{MC} 33 \mathrm{C} 35 \mathrm{cor}}=$ média de coeficiente de digestibilidade da MS com $\mathrm{C}_{33}+\mathrm{C}_{35}$ corrigida.

2 Médias seguidas de letras diferentes na coluna diferem $(P<0,05)$ pelo teste $\mathrm{t}$

${ }^{3} \mathrm{CV}(\%)=$ coeficiente de variação.

${ }^{4} \mathrm{DM}=$ diferença média entre coeficiente de digestibilidade aparente e estimada pelos alcanos.

${ }^{5} \mathrm{EP}=$ erro-padrão da média $(\mathrm{n}=20)$.

${ }^{1} C D M S_{A P}=$ coefficient of apparent $D M$ digestibility, $C D M S_{C_{33}}=$ coefficient of $D M$ digestibility estimated by $C_{33}, C D M S_{C 35}=$ coefficient of $D M$ digestibility estimated by $C_{35}$ ${ }_{C D M S_{M C 33 C 35}}$ a average coefficient of $D M$ digestibility estimated by $C_{33}+C_{35}$, $C D M S_{C 33 c o r}=$ coefficient of $D M$ digestibility estimated by $C_{33}$ corrected for fecal recovery, $C D M S_{C 3500}=$ coefficient of $D M$ digestibility estimated by $C_{35}$ corrected for fecal recovery, $C D M S_{M C 33 C 35 \text { cor }}=$ average coefficient of $D M$ digestibility estimated by $C_{33}+C_{35}$ corrected for fecal recovery.

${ }^{2}$ Means, within a row, followed by different letters differ $(P<0.05)$ by $t$ test.

${ }^{3} \mathrm{CV}=$ coefficient of variation.

${ }^{4} \mathrm{MD}=$ mean difference between measured and estimated ( $n$-alkanes) $D M$ digestibility.

${ }^{5} \mathrm{SE}=$ standard error $(n=20)$
Os valores da digestibilidade da MS foram subestima$\operatorname{dos}(\mathrm{P}<0,05)$ em 8,$65 ; 6,46$ e 7,55 pontos percentuais quando utilizados, respectivamente, os alcanos $\mathrm{C}_{33}$ e $\mathrm{C}_{35}$ e sua soma, sem correção para recuperação fecal. Ressalta-se que a magnitude das diferenças entre os valores reais e os estimados foi inversamente proporcional à taxa de recuperação fecal dos alcanos. Sempre que a recuperação fecal é incompleta, inferior a $100 \%$, a digestibilidade é subestimada. Como demonstrado na Tabela 2, as recuperações dos alcanos $\mathrm{C}_{33}$ e $\mathrm{C}_{35}$ foram inferiores a 89,45 e $92,70 \%$, respectivamente, comprovando a necessidade da correção para a recuperação fecal.

$\mathrm{O}$ uso dos alcanos corrigidos para recuperação fecal possibilitou valores mais acurados $(\mathrm{P}<0,05)$, não sendo observadas diferenças significativas $(P>0,05)$ entre os alcanos utilizados (Tabela 3). Entretanto, o uso do alcano $\mathrm{C}_{33}$ corrigido resultou em estimativas com menor coeficiente de variação $(5,36 \%)$, indicando que, embora possa ser utilizado qualquer um dos alcanos, desde que corrigidos para recuperação fecal, alcanos de maior concentração são mais indicados por apresentarem menores variações nas fezes e no processo analítico.

Comparado métodos da digestibilidade aparente pela coleta total e com o alcano $\mathrm{C}_{35}$ em três espécies forrageiras, Oliveira et al. (2000) obtiveram subestimativa da digestibilidade em 4,68\% (sem correção para recuperação fecal) com o uso do alcano $\mathrm{C}_{35}$ em relação à coleta total. Sandberg et al. (2000), estudando a digestibilidade da MS em bovinos com uso de indicadores internos (FDAI - fibra em detergente ácido indigestível e alcanos), encontraram coeficientes subestimados para os dois indicadores em relação à digestibilidade aparente pela coleta total.

$\mathrm{O}$ alcano $\mathrm{C}_{35}$ ou de cadeia maior, foi recomendado por Mayes (1999) para estimativas da digestibilidade e, principalmente, para casos em que a recuperação fecal dos alcanos é desconhecida. Entretanto, diversas espécies forrageiras tropicais apresentam baixas concentrações desses alcanos e, neste caso, recomenda-se utilizar alcanos de cadeia menor $\left(\mathrm{C}_{29}, \mathrm{C}_{31}\right.$ e $\left.\mathrm{C}_{33}\right)$ corrigidos para a recuperação fecal (Laredo, 1991).

Os valores do consumo real e estimado pelos $n$-alcanos, seus coeficientes de variação e as diferenças nas estimativas (consumo real menos consumo estimado) são apresentados na Tabela 4. Pela técnica dos alcanos naturais $\mathrm{C}_{31}$ e $\mathrm{C}_{33}$, verificou-se diferença significativa $(\mathrm{P}<0,05)$ pelo teste $\mathrm{t}$ entre consumo real e o estimado, que não diferiram $(\mathrm{P}>0,05)$ quando utilizada a média dos alcanos $\mathrm{C}_{31}$ e $\mathrm{C}_{33}$. O uso do alcano $\mathrm{C}_{31}$ subestimou em $13,31 \%$ o consumo de MS, ao passo que o alcano $\mathrm{C}_{33}$ superestimou em $9,06 \%$. O uso 
Tabela 4 - Valores médios dos consumos real e estimado pela técnica de $n$-alcanos utilizando-se os alcanos naturais $\mathrm{C}_{31}$ e $\mathrm{C}_{33}$ e a média do $\mathrm{C}_{31}+\mathrm{C}_{33}$

Table 4 - Means values of observed intake and intake estimated by the $n$-alkanes $C_{31}, C_{33}$ and the average of $C_{31}+C_{33}$

\begin{tabular}{|c|c|c|c|c|}
\hline $\begin{array}{l}\text { Item }^{1} \\
\text { Item }\end{array}$ & $\begin{array}{c}\text { Consumo médio } \\
\text { (kgMS/dia) } \\
\text { Intake } \\
\text { (kg DM/day) }\end{array}$ & $\mathrm{CV}(\%)^{2}$ & $\begin{array}{c}\mathrm{DM} \\
(\mathrm{kgMS} / \mathrm{dia})^{3} \\
M D \\
(\mathrm{~kg} D M / \text { day })\end{array}$ & $\begin{array}{c}\text { Erro médio } \\
\text { estimado }(\%)^{4} \\
\text { Estimated } \\
\text { error }(\%)^{4}\end{array}$ \\
\hline
\end{tabular}

\begin{tabular}{llccc}
\hline Real & $0,894^{\mathrm{b}}$ & 18,23 & - & - \\
Observed & & & & \\
$\mathrm{C}_{31} / \mathrm{C}_{32}$ & $0,775^{\mathrm{c}}$ & 32,00 & $0,119^{\mathrm{a}}$ & $13,31^{\mathrm{a}}$ \\
$\mathrm{C}_{33} / \mathrm{C}_{32}$ & $0,975^{\mathrm{a}}$ & 33,85 & $-0,081^{\mathrm{b}}$ & $-9,06^{\mathrm{b}}$ \\
$\mathrm{C}_{31}{ }^{+} \mathrm{C}_{33} / \mathrm{C}_{32}$ & $0,875^{\mathrm{b}}$ & 29,94 & $0,019^{\mathrm{c}}$ & $2,13^{\mathrm{c}}$ \\
$\mathrm{EP}^{5}$ & 0,019 & - & 0,018 & 1,88 \\
$\mathrm{SE}^{5}$ & & & &
\end{tabular}

$S E^{5}$

${ }_{1}^{1}$ Médias seguidas de letras diferentes na coluna diferem $(P<0,05)$ pelo teste $\mathrm{t}$ (Means, within a row, followed by different letters differ $[P<0.05]$ by $t$ test).

${ }^{2} \mathrm{CV}=$ coeficiente de variação (coefficient of variation).

${ }^{3} \mathrm{DM}=$ diferença média entre consumo real e estimado $(M D=$ mean difference between observed and estimated intakes).

4 Porcentagem de erro estimado em relação ao consumo real(Errorpercentage estimated in relation to the observed intake).

${ }^{5}$ Erro-padrão (Standard error).

simultâneo dos alcanos $\mathrm{C}_{31}$ e $\mathrm{C}_{33}$ não resultou em diferenças $(\mathrm{P}>0,05)$ para o consumo real.

$\mathrm{O}$ uso conjunto dos alcanos $\mathrm{C}_{31}$ e $\mathrm{C}_{33}$ possibilitou estimativa mais precisa, visto que seu coeficiente de variação $(29,94 \%)$ foi menor se comparado aos obtidos quando estes alcanos foram utilizados separadamente $\left(\mathrm{C}_{31}=32,00 \%\right.$ e $\mathrm{C}_{33}=33,85 \%$ ) (Tabela 4$)$. Na estimativa utilizando-se o alcano isoladamente, o uso do alcano interno adjacente $\left(\mathrm{C}_{33}\right)$ ao administrado $\left(\mathrm{C}_{32}\right)$ promoveu coeficiente mais acurado $(\mathrm{P}<0,05)$ e com precisão pouco menor (coeficiente de variação semelhante) que o obtido com $\mathrm{C}_{31}$. A estimativa pelo $\mathrm{C}_{33}$ foi mais acurada por possibilitar recuperação fecal mais próxima ao administrado $\left(1,22 \%\right.$ vs $3,25 \%$ para $\left.\mathrm{C}_{31}\right)$. A precisão na estimativa do consumo pode ser atribuída à curva de excreção fecal (Figura 1) do $\mathrm{C}_{33}$, que apresentou variação diurna maior que a de $\mathrm{C}_{31}$. Entretanto, a precisão da estimativa pode ser influenciada por outros fatores, como frequiência do fornecimento do indicador, erros durante a amostragem da dieta e das fezes, erros laboratoriais e erros na dosagem do indicador (variação na concentração das cápsulas).

A importância da mensuração da quantidade exata de indicador administrado foi comentada por Moore et al. (1992), citados por Detmann et al. (2001), pois afeta diretamente a acurácia e precisão das estimativas. Dove et al. (1989), comparando diferentes formas de administração do alcano externo (com papel filtro e pó de celulose em cápsulas de gelatina), em diferentes freqüências, não encontraram diferenças significativas na concentração fecal do alcano dosado. Mayes \& Duncan (1999) recomendaram utilizar
Tabela 5 - Consumo real e estimado pela técnica de alcanos utilizando-se a média dos alcanos $\mathrm{C}_{31}+\mathrm{C}_{33}(\mathrm{kgMS} / \mathrm{dia})$

Table 5 - Observed intake and intake estimated by the average combination of the alkanes $C_{31}+C_{33}$ ( $\left.k g D M / d a y\right)$

\begin{tabular}{lcccrr}
\hline $\begin{array}{l}\text { Itens } \\
\text { Item }\end{array}$ & $\begin{array}{c}\text { Consumo } \\
\text { médio real } \\
\text { Observed } \\
\text { intake }\end{array}$ & $\begin{array}{c}\text { Consumo } \\
\text { médio estimado } \\
\text { Estimated } \\
\text { intake }\end{array}$ & ${\mathrm{CV}(\%)^{2}}^{2}$ & $\begin{array}{r}\mathrm{DM}^{3} \\
(\%)^{4} \\
M D\end{array}$ & $\begin{array}{r}\text { Diferença } \\
\text { Difference }\end{array}$ \\
\hline 09:00 & 0,894 & 0,924 & 26,30 & $-0,030$ & $-3,36$ \\
$13: 00$ & 0,894 & 0,876 & 26,14 & 0,018 & 2,01 \\
$17: 00$ & 0,894 & 0,855 & 31,11 & 0,039 & 4,36 \\
$21: 00$ & 0,894 & 0,850 & 31,88 & 0,044 & 4,92 \\
$01: 00$ & 0,894 & 0,884 & 34,95 & 0,010 & 1,12 \\
$05: 00$ & 0,894 & 0,832 & 32,69 & 0,062 & 6,94 \\
$2^{5}$ & 0,894 & 0,900 & 29,88 & $-0,006$ & $-0,67$ \\
$3^{6}$ & 0,894 & 0,907 & 31,97 & $-0,013$ & $-1,45$ \\
$6^{7}$ & 0,894 & 0,850 & 27,88 & 0,044 & 4,92 \\
$\mathrm{EP}^{8}$ & 0,037 & 0,060 & - & 0,049 & 5,19 \\
$S P$ & & & & & \\
\hline
\end{tabular}

${ }^{1}$ Não-significativo $(P>0,05)$ pelo teste $t$ (Not significant $(P>0.05)$ by $t$ test).

$2 \mathrm{CV}(\%)=$ coeficiente de variação (Coefficient of variation).

$3 \mathrm{DM}=$ diferença média entre consumo real e estimado (kgMS/dia) (MD= mean difference between observed and estimated intake (kg DM/day).

4 Porcentagem de erro estimado em relação ao consumo real (\%) (Error percentage estimated in relation to the observed intake (\%)).

52 coletas (2 samplings): $(09: 00+21: 00 \mathrm{~h})$.

63 coletas (3 samplings): $(09: 00+17: 00+01: 00 \mathrm{~h})$.

76 coletas (6 samplings): $(09: 00+13: 00+17: 00+21: 00+01: 00+05: 00 \mathrm{~h})$.

8 Erro-padrão (Standard error).

péletes de papel-celulose picado, pois esta técnica oferece melhores estimativas, mesmo quando dosado uma vez ao dia.

Dados da literatura (Mayes et al. 1986; Dove \& Mayes, 1991; Reeves et al., 1996; Berry et al. 2000) comprovam a importância do uso do alcano $\mathrm{C}_{33}$ nas estimativas do consumo, visto que este alcano possibilita estimativas mais precisas por apresentar recuperação fecal semelhante ao administrado. Dove \& Mayes (1999) comentaram que o uso de alcanos com baixas concentrações na dieta representa importantes fontes de erro. Esses autores enfatizaram a importância de amostragens representativas das pastagens consumidas e do uso do alcano natural e do administrado com recuperações fecais semelhantes.

Os valores do consumo real e estimado pela técnica de $n$-alcanos, seus coeficientes de variação, as diferenças nas estimativas e os erros médios nas estimativas, por horário e por número de coletas, para o uso simultâneo dos alcanos $\mathrm{C}_{31}$ e $\mathrm{C}_{33}$, são apresentados na Tabela 5 .

Não houve diferença $(P>0,05)$ entre o consumo real e estimado nos diversos horários e número de coletas realizadas. Entretanto, coletas de fezes realizadas às 9 ou às $13 \mathrm{~h}$ resultaram em menores coeficientes de variação. Es ses horários coincidiram com o período de menor variação na concentração fecal dos alcanos internos e externos utilizados nos cálculos (Figura 1). 
Estudando estratégias de amostragem de fezes em ovinos, Vulich \& Hanrahan (1995) compararam o consumo utilizando a coleta total de fezes, o número de amostragens diárias realizadas diretamente no reto do animal e os dias de amostragens. Os autores verificaram que as estimativas de consumo pela coleta total não são influenciadas pelo número de amostragens realizadas diretamente do reto do animal. Entretanto, verificou-se que a variação se tornou maior à medida que aumentaram o número diário de coletas e o número de dias de coleta.

Segundo Detmann et al. (2001), em situações de pastejo, o fornecimento do indicador e a coleta de fezes exigem a contenção do animal, o que ocasiona estresse no animal, alterando o comportamento de pastejo, o consumo e, conseqüentemente, a excreção fecal. Assim, quanto menor o número de dias de coleta e o número de coletas durante o dia (desde que não comprometa as estimativas), menor a interferência no comportamento do animal em pastejo, gerando resultados mais exatos.

\section{Conclusões}

As concentrações dos alcanos internos presentes no feno de Brachiaria decumbens e no feno de amendoim forrageiro permitem estimar o consumo e a digestibilidade em ovinos.

Para estimativas de melhor qualidade da digestibilidade da MS, deve-se utilizar o alcano interno $\mathrm{C}_{33}$ corrigido para recuperação fecal.

Para estimar o consumo de MS, devem-se utilizar de forma conjunta nos cálculos os alcanos internos $C_{31} \mathrm{e}$ $\mathrm{C}_{33}$, sem necessidade de correção para recuperação fecal.

Estimativas adequadas do consumo de matéria seca podem ser obtidas a partir de uma amostra de fezes por animal por dia, colhida pela manhã (9h) ou à tarde (13h). Amostragens suplementares não melhoram a qualidade das estimativas.

\section{Literatura Citada}

BERRY, N.R.; SCHEEDER M.R.L.; SUTTER, F. et al. The accuracy of intake estimation based on the use of alkane controlledrelease capsules and faeces grab sampling in cows. Annales de Zootechnie, v.49, p.3-13, 2000.

CHEN, W.; LEFROY, R.D.B.; SCOTT, J.M. et al. Field variations in alkane signatures among plant species in 'degraded' and perennial pastures on the Northern Tablelands of New South Wales. Australian Journal of Agriculture Research, v.49, n. 8, p.263-268, 1998 .

CÔRTES, C.; DAMASCENO, J.C.; BÉCHET, G. et al. Species composition in ryegrass (Lolium perenne)/tall fescue (Festuca arundinacea) mixtures using various combinations of $n$-alkanes. Grass and Forage Science, v.60, p.254-261, 2005.
CÔRTES, C.; DAMASCENO, J.C.; FUKUMOTO, N.M. et al. Potencial discriminatório dos $\mathrm{N}$-alcanos em plantas forrageiras tropicais por análises multivariadas. Revista Brasileira de Zootecnia, v.34, n.4, p.1079-1087, 2005a.

CÔRTES, C.; DAMASCENO, J.C.; PAINE, R.C. et al. Uso de $n$-alcanos na estimativa da composição botânica em amostras com diferentes proporções de Brachiaria brizantha e Arachis pintoi. Revista Brasileira de Zootecnia, v.34, n.5, p.1468-1474, 2005b.

DETMANN, E.; PAULINO, M.F.; ZERVOUDAKIS, J.T. et al. Cromo e indicadores internos na determinação do consumo de novilhos mestiços, suplementados, a pasto. Revista Brasileira de Zootecnia, v.30, n.5, p.1600-1609, 2001.

DOVE, H. Using the $n$-alkanes of plant cuticular wax to estimate the species composition of herbage mixtures. Australian Journal of Agricultural Research, v.43, p.1711-1724, 1992.

DOVE, H.; MAYES, R.W.; FREER, M. et al. Faecal recoveries of the alkanes of plant cuticular waxes in penned and in grazing sheep. In: INTERNATIONAL GRASSLANDS CONGRESS, 16. 1989, Nice. Proceedings... Nice: International Grassland Society, 1989. p.1093-1094.

DOVE, H.; MAYES, R.W. The use of plant wax alkanes as marker substances in studies of the nutrition of herbivores: a review. Australian Journal of Agriculture Research, v.42, p.913-952, 1991

EUCLYDES, R.F. SAEG - Sistema de análises estatísticas e genéticas (Manual do usuário). Viçosa, MG: Universidade Federal de Viçosa, 1993. 59p.

LAREDO, M.A.; SIMPSON, D.J.; ORPIN, C.G. The potential for using $n$-alkanes in tropical forages as a marker for the determination of dry matter by grazing ruminants. Journal Agricultural Science, v.117, p.355-361, 1991.

MACEDO, V.P.; DAMASCENO, J.C.; SANTOS, G.T. et al. Comportamento da curva de lactação de cabras mestiças saanen em função da suplementação de concentrado e do sistema de produção. Revista Brasileira de Zootecnia, v.30, p.2093-2098, 2001

MARAIS, J.P.; FIGENSCHOU, D.L.; ESCOTT-WATSON, P.L. et al. Administration in suspension-form of alkane external markers for dry matter intake and diet selection studies. Journal of Agricultural Science, v.126, p.207-210, 1996.

MAYES, R.W. The potential of plant-wax compounds as markers for digestion and utilization in free-ranging herbivores. In: INTERNATIONAL SYMPOSIUM NUTRITION OF HERBIVORES, 5., 1999, San Antonio. Proceedings... San Antonio: [1999]. (CD-ROM).

MAYES, R.W.; DUNCAN, A.J. New developments in the use of plant-wax markers to determine intake. In: INTERNATIONAL SYMPOSIUM NUTRITION OF HERBIVORES, 5., 1999, San Antonio. Proceedings... San Antonio: [1999]. (CD-ROM).

MAYES, R.W.; LAMB, C.S. The possible use of n-alkanes in herbage as indigestible faecal markers. Proceedings of the Nutritional Society, v.49, p.39, 1984. (abst.).

MAYES, R.W.; LAMB, C.S; GOLGROVE, P.M. Digestion and metabolism of dosed even-chain and herbage odd-chain n-alkanes in sheep. In: GENERAL MEETING EUROPEAN GRASSLANDS FEDERATION, 12., 1988, Dublin. Proceedings... Dublin: European Grasslands Federation, 1988. p.159-63.

MAYES, R.W.; LAMB, C.S; GOLGROVE, P.M. The use of herbage $n$-alcanes as markers for the determination of herbage intake. Journal Agricultural Science, v.107, p.161-170, 1986.

NEWMAN, J.A.; THOMPSON, W.A.; PENNING, P.D. Leastsquares estimation of diet composition from $n$-alkanes in herbage and faces using matrix mathematics. Australian Journal of Agriculture Research, v.46, p.793-805, 1995.

OLIVEIRA, D.E. Uso da técnica de $n$-alcanos para medir o aporte de nutrientes através de estimativas do consumo de forragem em bovinos. Piracicaba: Escola Superior de Agricultura "Luiz de Queiroz", 2002. 129p. Tese (Doutorado 
em Agronomia) - Escola Superior de Agricultura "Luiz de Queiroz", 2002.

OLIVEIRA, D.E.; PRATES, E.R.; PERALBA, M.C.R. Digestibilidade determinada pelo método indireto usando o $n$-alcano $\mathrm{C}_{35}$. Revista Brasileira de Zootecnia, v.29, n.3, p.848-852, 2000.

REEVES, M.; FULKERSON, W.J.; KELLAWAY, R.C. et al. A comparison of three techniques to determine the herbage intake of dairy cows grazing kikuyu (Pennisetum clandestinum) pasture. Australian Journal of Experimental Agriculture, v.36, p.23-30, 1996.

SANDBERG, R.E.; ADAMS, D.D.; KLOPFENSTEIN, T.J. et al. [2000]. $\boldsymbol{N}$-alkane as an internal marker for predicting digestibility of forages. Disponível em: http://uvalde.tamu. edu/jrm/mar00/sandberg.htm. Acesso em: 03/12/01.

SILVA, P.R.C.; NASCIMENTO JR., D. [2001]. Aspecto do consumo em ruminantes sob pastejo. Disponível em: www.tdnet.com.br/ domicio/aspectos.htm. Acesso em: 20/08/01.
VULICH, S.A.; HANRAHAN, J.P. Faecal sampling for the estimation for herbage intake using $n$-alkanes: evaluation of sample pooling and the use of rectal grab samples. Journal of Agricultural Science, v.124, p.79-86, 1995.

VULICH, S.A.; HANRAHAN, J.P.; CROWLEY, B.A. Modification of the analytical procedures for the determination of herbage and fecal n-alkanes used in the estimation of herbage intake. Journal of Agricultural Science, v.124, p.71-77, 1995.

WILLIANS, J.C. An empirical model for the lactation curve of white British dairy goats. Animal Production, v.57, p.91-97, 1993.

Recebido: 17/11/05 